\title{
Clinicians' abilities to estimate cardiac index in ventilated children and infants
}

\author{
Shane M Tibby, Mark Hatherill, Michael J Marsh, Ian A Murdoch
}

\begin{abstract}
Objectives-To evaluate the ability of clinicians involved in the provision of paediatric intensive care to estimate cardiac index in ventilated children, based on physical examination and clinical and bedside laboratory data.

Methods-Clinicians were exposed to all available haemodynamic and laboratory data for each patient, allowed to make a physical examination, and asked to first categorise cardiac index as high, high to normal, low to normal, or low, and then to quantify this further with a numerical estimate. Cardiac index was measured simultaneously by femoral artery thermodilution (coefficient of variation $5.37 \%$ ). One hundred and twelve estimates were made by 27 clinicians on 36 patients (median age 34.5 months).

Results-Measured cardiac index ranged from 1.39 to $6.841 / \mathrm{min} / \mathrm{m}^{2}$. Overall, there was poor correlation categorically ( $\kappa$ statistic 0.09 , weighted $\kappa 0.169$ ) and numerically $(r=0.24,95 \%$ confidence interval 0.06 to 0.41 ), although some variation was seen among the various levels of seniority. Conclusion-Assuming that objective measurement, and hence manipulation, of haemodynamic variables may improve outcome, these findings support the need for a safe, accurate, and repeatable technique for measurement of cardiac index in children who are critically ill.

(Arch Dis Child 1997;77:516-518)
\end{abstract}

Keywords: cardiac index; thermodilution

It is some 25 years since the development of the pulmonary artery thermodilution catheter for bedside measurement of cardiac output, which allows calculation of oxygen delivery and consumption. The measurement and manipulation of these variables figure prominently in current practice in adult intensive care units; this is not the case, however, in many paediatric units in the UK. In a recent survey of 24 paediatric intensive care units (those containing more than four beds), only one third had access to thermodilution for measurement of cardiac output, with only 40 pulmonary artery catheters being used in the year surveyed. ${ }^{1} \mathrm{~A}$ variety of methods are available for measuring cardiac output in the paediatric population, ${ }^{2}{ }^{3}$ but there may be several reasons for their limited use, including technical constraints, cost, perceived usefulness, complications and difficulties of central vascular access, and the belief that cardiac output can be clinically estimated in children. ${ }^{4}$ As a result, many paediatric clinicians in intensive care units rely on indirect variables of cardiac performance (heart rate, blood pressure, pulse volume, central venous pressure) and organ perfusion (urine output, capillary refill, core-peripheral temperature difference, mental state, serum lactate concentration, and base excess) when manipulating treatment regimens.

Clinical estimation of cardiac output, and hence cardiac index (cardiac index = cardiac output divided by body surface area), in adult practice has been shown to be inaccurate,$^{56}$ and may promote suboptimal or even inappropriate interventions. ${ }^{7-9}$ We wanted to investigate whether similar inaccuracies exist in clinicians' abilities to estimate cardiac index in ventilated children.

\section{Patients and methods}

Estimates of cardiac index were made on ventilated patients in whom objective measurement of cardiac index by femoral artery thermodilution (COLD Z-021, Pulsion Medical Systems, Munich, Germany) was undertaken for clinical reasons.

One hundred and twelve estimates of cardiac index were made by 27 clinicians on 36 patients, median age 34.5 months (interquartile range 4.8-90.8 months), median weight $12.9 \mathrm{~kg}$ (range $2.5-64 \mathrm{~kg}$ ). Patient diagnoses were: after cardiac surgery (with no residual shunt) ( $\mathrm{n}=27)$, meningococcal sepsis $(\mathrm{n}=4)$, staphylococcal sepsis $(n=2)$, acute respiratory distress syndrome $(n=1)$, nephrotic syndrome $(\mathrm{n}=1)$, and acute life threatening event $(\mathrm{n}=$ 1). All patients were ventilated and sedated. Clinicians originated from a variety of paediatric subspecialties, including intensive care, cardiology, and anaesthetics, and ranged from consultant to senior house officer level. Before estimating cardiac index, clinicians were exposed to all clinical and laboratory data available on the patients, and were allowed to make a physical examination. They were initially asked to estimate the category of cardiac index (high $\geqslant 51 / \mathrm{min} / \mathrm{m}^{2}$, high to normal 4.0-4.9, low to normal 3.0-3.9, and low $<3.0$ ) and then the absolute value. Concurrently, five consecutive femoral artery thermodilution measurements were made and averaged.

Thermodilution involved a central venous injection of cold $\left(<10^{\circ} \mathrm{C}\right) 5 \%$ dextrose of appropriate volume for the patient's weight $(1.5 \mathrm{ml}+0.15 \mathrm{ml} / \mathrm{kg})$, sensed by a 1.3 French thermistor placed into the descending aorta via 


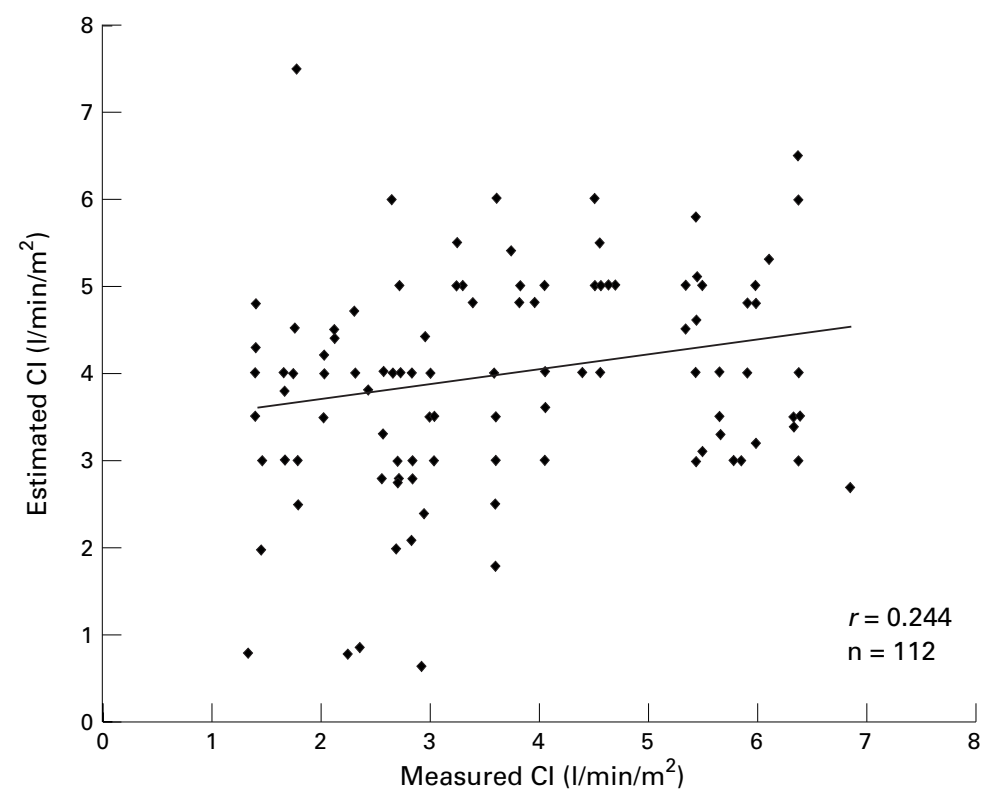

Figure 1 Estimated v measured cardiac index (CI) from all grades.

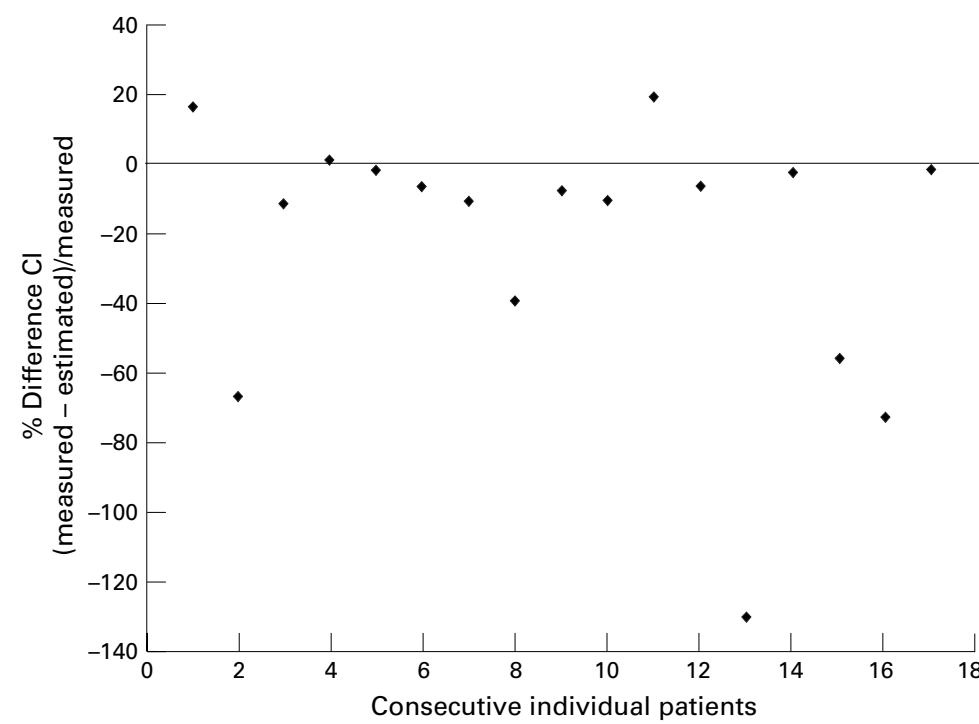

Figure 2 Percentage discrepancy of estimated cardiac index (CI) by thermodilution clinician before measurement on consecutive patients.

Table $1 \kappa$ Statistic for estimated $v$ measured cardiac index (CI)

\begin{tabular}{llllll}
\hline$\kappa=0.09$ & \multicolumn{3}{l}{ Estimated CI } & \\
\cline { 2 - 5 } Weighted $\kappa=0.169$ & Low & Low to normal & High to normal & High & \multirow{2}{*}{ Total } \\
\cline { 2 - 5 } & & & & & \\
Measured CI & 12 & 13 & 17 & 5 & 47 \\
$\quad$ Low & 3 & 5 & 5 & 6 & 19 \\
Low to normal & 0 & 2 & 4 & 7 & 13 \\
$\quad$ High to normal & 1 & 12 & 8 & 12 & 33 \\
$\quad$ High & 16 & 32 & 34 & 30 & 112 \\
Total & &
\end{tabular}

Strength of agreement for $\kappa:<0.20$ poor; $0.21-0.40$ fair; $0.4-0.60$ moderate; $0.61-0.80$ good; $0.81-1.00$ very good. values of cardiac index and the $\kappa$ statistic was calculated for categorical assessment. ${ }^{11}$ The coefficient of variation quantified the repeatability of femoral artery thermodilution. As the study did not involve alteration in patient management, ethical approval was not sought.

\section{Results}

Measured cardiac index ranged from 1.39 to $6.84 \mathrm{l} / \mathrm{min} / \mathrm{m}^{2}$ (coefficient of variation $5.37 \%$ ). Overall, there was poor correlation (fig 1) between absolute measured and estimated cardiac index: $r=0.24$ (95\% confidence interval 0.06 to $0.41, \mathrm{n}=112$ ). Variation was seen among the levels of seniority: consultants $r=$ $0.19(-0.23$ to $0.54, \mathrm{n}=25)$, fellows $r=0.38$ (0.12 to $0.58, \mathrm{n}=56)$, senior house officers $r=$ $0.04(-0.32$ to $0.39, \mathrm{n}=31)$, 'thermodilution clinician' $r=0.82(0.56$ to $0.93, \mathrm{n}=17)$. For categorical assessment, $\kappa=0.09$ and weighted $\kappa=0.169$ (see table 1). Both indicate a poor strength of agreement. Figure 2 shows the percentage inaccuracy of the measuring clinician's estimates with time.

\section{Discussion}

Our results show clinicians' inaccuracies in estimating cardiac index in children. Futhermore, these inaccuracies appear to be spread across disciplines and levels of seniority. Similar inaccurate predictions of haemodynamic profiles have been found in adult intensive care unit practice, with invasive monitoring promoting changes in treatment from $45-58 \%$ of the time..$^{5-9}$ Whether measurement of cardiac index has a beneficial effect on mortality in the adult population is questionable, however. ${ }^{72}$ To our knowledge, this question has yet to be adequately studied in children. Although a high cardiac index does not necessarily imply an improved outcome, ${ }^{13}$ it would be fair to speculate that an increase in cardiac index in those patients in whom flow is low and inadequate, increases the chance of survival. Our data also show that although positive reinforcement played a part in one clinician's estimating ability, rather large discrepancies occasionally arose, and this did not improve with time. Two aspects were not examined in this study: firstly, whether clinicians could quantify changes in cardiac output, and secondly, whether invasive monitoring resulted in changes in treatment and outcome. Given the poor strength of agreement when categorising cardiac index, shown by a $\kappa$ statistic of 0.09 , it is unlikely that clinicians would be able to accurately quantify changes in cardiac index. The question of whether invasive monitoring results in significant changes in treatment and outcome was beyond the scope of this study. Certainly, optimising haemodynamic variables to 'ideal' supranormal values in adults have not been shown to improve outcome when applied to whole patient populations, ${ }^{14}{ }^{15}$ but this does not necessarily mean that cardiac index and other variables should not be optimised to meet the metabolic needs of individual patients. This same logic can apply to less invasive monitoring procedures such as pulse oximetry. It has been shown that monitoring with pulse 
oximetry in the perioperative period does not reduce overall complications, yet this continues to be a standard monitoring procedure. ${ }^{16} \mathrm{We}$ would suggest that measurement of cardiac index should not be taken as an isolated variable, but rather used as part of the overall assessment of myocardial performance in conjunction with other modalities. For example, echocardiography may show up abnormalities such as diastolic dysfunction or segmental wall abnormalities which are not readily apparent on flow measurement alone. Reluctance on the part of paediatricians to measure cardiac index in children may be for several reasons: that traditional methods have a not insignificant complication rate, that obtaining access is often a problem (especially in the very young), and the lack of a yardstick makes validation of new techniques difficult. The wisdom of wide spread use of the pulmonary artery catheter has recently been questioned. ${ }^{17}$ With the advent of newer less invasive technologies, however, and refinement of existing methods such as the transoesophageal Doppler, ${ }^{18}$ thoracic bioimpedance, ${ }^{19}$ femoral artery thermodilution, ${ }^{10}$ and direct Fick via indirect calorimetry, ${ }^{20}$ monitoring of cardiac index in children will become a realistic option in most intensive care units.

1 Murdoch I. Measurement of cardiac output in children: a United Kingdom perspective. Clinical Intensive Care 1994;4(suppl 5):29.

2 Fanconi S, Burger R. Measurement of cardiac output in children. Intensive Care World 1992;9:8-12.

3 Murdoch IA, Marsh MJ, Morrison G. Measurement of cardiac output in children. In: Vincent J-L, ed. 1995 yearbook of intensive care and emergency medicine. Berlin: Springer, 1995:606-14.

4 Smith-Wright DL, Green TP, Lock JE, Egar MI, Fuhrman BP. Complications of vascular catheterization in critically ill children. Crit Care Med 1984;12:1015-7.
5 Eisenberg PR, Jaffe AS, Schuster DP. Clinical evaluation compared to pulmonary artery catheterization in the hemodynamic assessment of critically ill patients. Crit Care Med 1984;12:549-53.

6 Connors AF, McCaffree DR, Gray BA. Evaluation of rightheart catheterization in the critically ill patient without acute myocardial infarction. N Engl f Med 1983;308:263-7.

7 Mimoz O, Rauss A, Rekik N, Brun-Buisson C, Lemaire F, Brochard L. Pulmonary artery catheterization in critically ill patients: a prospective analysis of outcome changes associated with catheter-prompted changes in therapy. Crit Care Med 1994;22:573-9.
Cared with catheter-promp

8 Steingrub JS, Celoria G, Vickers-Lahti M, Teres D, Bria W. Therapeutic impact of pulmonary artery catheterization in a medical/surgical ICU. Chest 1991;99:1451-5.

9 Celoria G, Steingrub JS, Vickers-Lahti M, et al. Clinical assessment of hemodynamic values in two surgical intensive care units. Arch Surg 1990;125:1036-9.

10 McLuckie A, Murdoch IA, Marsh MJ, Anderson D. A comparison of pulmonary and femoral artery thermodilution parison of pulmonary and femoral artery thermodilution
cardiac indices in paediatric intensive care patients. Acta cardiac indices in paediati
Paediatr 1996;85:336-8.

11 Altman DG. Practical statistics for medical research. London: Chapman and Hall, 1991:403-7.

12 Tuchschmidt J, Sharma P. Impact of hemodynamic monitoring in a medical intensive care unit. Crit Care Med 1987;15:840-3.

13 Pollack MM, Fields AI, Ruttimann UE. Distribution of cardiopulmonary variables in pediatric survivors of septic shock. Crit Care Med 1985;13:454-9.

14 Hayes MA, Timmins AC, Yau ES, Palazzo M, Hinds CJ, Watson D. Elevation of systemic oxygen delivery in the Watson D. Elevation of systemic oxygen delivery in the
treatment of critically ill patients. N Engl F Med 1994;330: treatment
$1717-22$

15 Gattinoni L, Brazzi L, Pelosi P, et al. A trial of goal-orientated hemodynamic therapy in critically ill patients. SvO2 collaborative group. $N$ Engl $f$ Med 1995;333:1025-32.

16 Moller JT, Johannessen NW, Espersen K, et al. Randomized evaluation of pulse oximetry in 20,802 patients: II. Perioperative events and postoperative complications. Perioperative events and posto
Anesthesiology 1993;78:445-53.

17 Connors AF, Speroff T, Dawson NV, et al. The effectiveness of right heart catheterization in the initial care of critically ill patients. $\mathcal{F} A M A$ 1996;276:889-97.

18 Murdoch IA, Marsh MJ, Tibby SM, McLuckie A. Continuous haemodynamic monitoring in children: use of transoesophageal Doppler. Acta Paediatr 1995;84:761-4.

19 O'Connell AJ, Tibballs J, Coulthard M. Improving agreement between thoracic bioimpedance and dye dilution cardiac output estimation in children. Anaesth Intensive Care 1991;19:434-40.

20 Weyland W, Weyland A, Fritz U, Redecker K, Ensink FB, Braun U. A new paediatric metabolic monitor. Intensive Care Medicine 1994;20:51-7. 